\title{
BioPATH: A Biomarker Study in Asian Patients with HER2+ Advanced Breast Cancer Treated with Lapatinib and Other Anti-HER2 Therapy
}

\author{
Sung-Bae Kim, MD, PhD' \\ In-Gu Do, MD, PhD² \\ Janice Tsang, $\mathrm{MD}^{3}$ \\ Tae-You Kim, MD, PhD ${ }^{4}$ \\ Yoon-Sim Yap, MBBS, FRACP, PhD 5 \\ Gerardo Cornelio, $\mathrm{MD}^{6}$ \\ Gyungyub Gong, $\mathrm{MD}, \mathrm{PhD}^{7}$ \\ Soonmyung Paik, MD \\ Suee Lee, $\mathrm{MD}, \mathrm{PhD}^{9}$ \\ Ting-Ying Ng, MD ${ }^{10}$ \\ Sarah Park, MD ${ }^{11}$ \\ Ho-Suk Oh, MD, PhD'2 \\ Joanne Chiu, $\mathrm{MBBS}^{3}$ \\ Joohyuk Sohn, MD, PhD ${ }^{13}$ \\ Moonhee Lee, MD, PhD ${ }^{14}$ \\ Young-Jin Choi, MD, PhD ${ }^{15}$ \\ Eun Mi Lee, MD ${ }^{16}$ \\ Kyong-Hwa Park, MD, PhD ${ }^{17}$ \\ Christos Nathaniel, PhD'18 \\ Jungsil Ro, MD, $\mathrm{PhD}^{19}$
}

${ }^{*} A$ list of author's affiliations appears at the end of the paper.

Correspondence: Jungsil Ro, MD, PhD Center for Breast Cancer, National Cancer Center, 323 Ilsan-ro, Ilsandong-gu,

Goyang 10408, Korea

Tel: 82-31-920-1680

Fax: 82-31-920-0450

E-mail: jungsro@ncc.re.kr

Received November 3, 2018

Accepted March 19, 2019

Published Online June 4, 2019

*Sung-Bae Kim and In-Gu Do contributed equally to this work.

*Presented at: San Antonio Breast Cancer Symposium; December 10-14, 2013; San Antonio, TX, USA (Poster P4-12-28), Kim SB et al. HER2-related biomarkers in HER2+ breast cancer patients in Asia Pacific.

\section{Purpose}

BioPATH is a non-interventional study evaluating the relationship of molecular biomarkers (PTEN deletion/downregulation, PIK3CA mutation, truncated HER2 receptor [p95HER2], and tumor HER2 mRNA levels) to treatment responses in Asian patients with HER2+ advanced breast cancer treated with lapatinib and other HER2-targeted agents.

\section{Materials and Methods}

Female Asian HER2+ breast cancer patients $(n=154)$ who were candidates for lapatinibbased treatment following metastasis and having an available primary tumor biopsy specimen were included. The primary endpoint was progression-free survival (PFS). Secondary endpoints were response rate, overall survival on lapatinib, correlation between biomarker status and PFS for any previous trastuzumab-based treatment, and conversion/conservation rates of the biomarker status between tissue samples collected at primary diagnosis and at recurrence/metastasis. Potential relationships between tumor mRNA levels of HER2 and response to lapatinib-based therapy were also explored.

\section{Results}

p95HER2, PTEN deletion/downregulation, and PIK3CA mutation did not demonstrate any significant co-occurrence pattern and were not predictive of clinical outcomes on either lapatinib-based treatment or any previous trastuzumab-based therapy in the metastatic setting. Proportions of tumors positive for p95HER2 expression, PIK3CA mutation, and PTEN deletion/down-regulation at primary diagnosis were $32 \%, 31.2 \%$, and $56.2 \%$, respectively. Despite limited availability of paired samples, biomarker status patterns were conserved in most samples. HER2 mRNA levels were not predictive of PFS on lapatinib.

\section{Conclusion}

The prevalence of p95HER2 expression, PIK3CA mutation, and PTEN deletion/downregulation at primary diagnosis were similar to previous reports. Importantly, no difference was observed in clinical outcome based on the status of these biomarkers, consistent with reports from other studies.
Key words

Biomarkers, Breast neoplasms, HER2, Lapatinib, Trastuzumab 


\section{Introduction}

In recent years, breast cancer has become an emerging health issue for Asian women [1-4]. Effective populationbased treatment planning and monitoring may reduce the rising concern and burden of breast cancer in Asia. Overexpression of human epidermal growth factor receptor 2 (HER2) has been observed in 15\% to 20\% of invasive breast cancers and is recognized to be a driving force of tumor biology [5]. Therapeutic strategies to block the HER2 signaling pathway include monoclonal antibodies and small-molecule tyrosine kinase inhibitors (TKI). In context of recent developments in our understanding of drug resistance mechanisms of breast cancer, there is a continuous effort to develop new effective therapies.

Trastuzumab is a standard treatment for HER2-positive (HER2+) breast cancer in both adjuvant and advanced settings. Clinical studies have demonstrated significantly prolonged time to disease progression, a higher rate of objective response, a longer duration of response, and increased survival when trastuzumab is added to chemotherapy in patients with previously untreated metastatic breast cancer (MBC) [6]. Furthermore, the addition of trastuzumab to chemotherapy significantly improved clinical outcome in patients in multiple clinical trials in the early and locally advanced settings [7-10]. Since the development of trastuzumab, advancements in HER2-targeting therapies have led to approval of three additional agents in the metastatic setting-pertuzumab, trastuzumab emtansine (T-DM1), and lapatinib. Key recommendations for first-line treatment of HER2+ advanced breast cancer (ABC) or MBC include combination of HER2 inhibitors trastuzumab and pertuzumab with a taxane (preferred) regardless of prior adjuvant trastuzumab, alone or with chemotherapy. T-DM1 (preferred approach) or other anti-HER2 treatments in combination with chemotherapy are recommended treatment options in the second line and beyond [11-13].

Lapatinib, a TKI targeting epidermal growth factor receptor and HER2, significantly prolonged time to progression in combination with capecitabine versus capecitabine alone in patients whose metastatic tumors were progressing on trastuzumab-based chemotherapy [14]. Lapatinib has also demonstrated significant clinical benefit in Asian patients with HER2 + ABC. A study primarily conducted in Asian patients $(86 \%)$ demonstrated that lapatinib plus paclitaxel offers a significantly improved overall survival (OS), progression-free survival (PFS), and overall response rate (RR) over paclitaxel alone in patients with HER2+ MBC [15]. Lapatinib plus capecitabine was well tolerated in Japanese patients with HER2 + ABC or MBC who had progressed on trastuzumab [16]. Results from two independent studies confirmed that the combination of lapatinib and capecitabine is an effective and well-tolerated treatment option in a population of Chinese women with HER2 + ABC or MBC who have had disease progression following other treatments $[17,18]$. Currently, lapatinib-based therapy is approved in several countries for the treatment of HER2+ ABC that progressed on treatment regimens containing an anthracycline, a taxane, and trastuzumab.

De novo and acquired resistance to HER2-directed approaches remains a clinical challenge. Despite advances in the management of metastatic HER2+ breast cancer, the RR in the first-line anti-HER2 therapy setting ranges from $50 \%$ to $80 \%$, and from only $20 \%$ to $40 \%$ in the second-line setting [6,14,19-22]. Many single-gene molecular markers have been evaluated as predictors of response to specific regimens; however, reliable molecular predictors for efficacy of antiHER2 therapies are lacking. A number of reports suggest the presence of amino-terminally-truncated HER2 (p95HER2) [23,24], PI3KCA somatic mutations [25], deletion/down-regulation of the phosphatase and tensin homolog (PTEN) gene [26], and tumor HER2 mRNA levels $[27,28]$ as potential markers of response or resistance to anti-HER2 therapy. Altered expression of p95HER2, PI3KCA, and PTEN is associated with impaired efficacy of trastuzumab and/or pertuzumab therapy in MBC [23,29-31], although T-DM1 has been reported to be effective in both wild-type and PIK3CAmutated tumors [32]. In Japanese patients with breast cancer receiving lapatinib monotherapy after trastuzumab-based therapies, biomarker analyses suggest clinical benefit in patients with tumors harboring PIK3CA mutation or low expression of PTEN $[33,34]$. Further research into these biomarkers may provide valuable insights into their reliability to predict the success or failure of specific anti-HER2 treatment strategies. Moreover, the availability of predictive biomarkers may help to guide a more cost-effective and rational use of anticancer drugs.

Tumors harboring p95HER2 are potential targets for lapatinib therapy because of lapatinib's ability to bind to the adenosine triphosphate binding site in the intracellular kinase domain of HER2; in contrast, trastuzumab binds an extracellular epitope on HER2, which is absent from truncated p95HER2. In a previous report [25], tumor progression was more likely among trastuzumab-treated subjects with PIK3CA mutations than those without. Interestingly, some biomarker studies suggested that lapatinib may have benefit in tumors that have achieved some level of escape from HER2 dependence through PIK3CA mutation [33,34]. Clinical efficacy of trastuzumab but not lapatinib was impaired in tumors with PTEN downregulation/deletion [35]. Thus all three biomarkers have the potential to be predictive of lapatinib's clinical utility in ABC. Limited availability of biomarker studies for lapatinib constrains our ability to predict 
the success or failure of lapatinib-based regimens for treatment of patients with HER2 $+\mathrm{ABC}$.

This non-interventional study investigated the frequency of biomarkers, including p95HER2 expression, PTEN deletion/ down-regulation, and PIK3CA mutation, in addition to HER2 mRNA levels in lapatinib-treated and/or trastuzumab-treated patients with breast cancer, and their use as predictive markers in the HER2 treatment paradigm.

\section{Materials and Methods}

\section{Study design}

BioPATH is an international multicenter, observational study designed to investigate the frequency of biomarkers, including p95HER2 expression, PTEN deletion/ down-regulation, and PIK3CA mutation, in female patients with HER2+ ABC treated with lapatinib-based therapy. Patients were included who received other HER2-targeted agents before study entry. Biomarker status was quantified in the analysis population, and possible relationships between biomarkers and outcomes, adjusting for other clinical and pathological factors, were measured. Patients were enrolled in 17 centers in five countries (Hong Kong, Korea, Malaysia, the Philippines, and Singapore). Data from the single center in Malaysia could not be verified, following the death of the principal investigator, and was not included $(\mathrm{n}=2)$. The local ethics review boards approved the protocol, and all patients gave written informed consent before study enrollment. Patients continued with their regular treatment during the study and no treatment interventions were made.

\section{Study endpoints}

The primary endpoint was PFS, defined as time (in weeks) from initiation of the first lapatinib-based regimen given in the metastatic setting to disease progression from that regimen or death from any cause. The correlation of p95HER2 expression, PTEN deletion/down-regulation, and PIK3CA mutation, individually and in combination, with PFS following lapatinib-based treatment. Secondary endpoints were RR and OS among females initiated on the lapatinib-based regimen, biomarker levels and their association with PFS on previous trastuzumab-based treatment in the metastatic setting (defined as time from initiation of the first trastuzumabbased regimen given in the metastatic setting to disease progression from that regimen or death from any cause), and biomarker conservation / conversion rates. The RR was defined as percentage of patients showing complete response (CR) or partial response (PR) to lapatinib-based treatment, based on physician's clinical judgment. The OS was defined as time (in weeks) from initiation of the first lapatinib-based regimen until death due to any cause. For patients who were alive at the end of study or lost to follow-up, time to death was censored at the time of last contact. Biomarker conversion/conservation rates between primary diagnosis and at recurrence/ metastasis stratified according to the treatment received before the re-biopsy were explored for paired samples. Exploratory analyses were performed to investigate potential relationships between HER2 tumor mRNA levels and response to lapatinib-based therapy. All enrolled patients were followed until death, loss to follow-up, or until data cutoff date, whichever came first. During the study, patients were managed as per physician's standard medical care.

\section{Patients}

Female patients with confirmed HER2+ metastatic/recurrent breast cancer who were exposed to $\leq 2$ lines of trastuzumab-based regimens in the metastatic setting prior to start of lapatinib-based regimen were eligible for this study. Patients who received systemic treatment for either early-stage or/ and recurrent/metastatic disease were eligible for enrollment. Patients needed to have a historical tumor biopsy specimen available from their primary breast cancer diagnosis, or at least an available specimen from the period before starting any anti-HER2 therapy. Prior exposure to other antiHER2 therapies (e.g., pertuzumab, T-DM1, neratinib, ertumaxomab, AV-412, BIBW2992, CUDC-101, and anti-HER2 vaccines) except trastuzumab was not allowed. Patients with primary lesions that were not of breast origin were also excluded.

\section{Assessment}

We performed biomarker analysis on p95HER2, PI3KCA, and PTEN to explore associations of these markers with clinical outcomes on either lapatinib-based or trastuzumabbased therapy in the metastatic setting. p95HER2, PTEN, and PIK3CA biomarker assays were performed at a central laboratory (Samsung Medical Center, Sungkyunkwan University School of Medicine, Seoul, Korea) on sections from formalinfixed, paraffin-embedded (FFPE) tumor blocks. p95HER2 expression was initially evaluated by immunohistochemistry (IHC) assay (by bioMérieux, but was later changed to the VeraTag quantitative assay technology) for determining p95HER2 expression (Monogram Biosciences, South San Francisco, CA) [36]. An IHC 3+ result was considered as HER2+. PTEN was assayed by IHC using a PTEN antibody (clone Y184, Abcam, Cambridge, UK) on a Dako Autostainer system (Dako, Glostrup, Denmark). The Sequenom MassAR- 
RAY platform (Sequenom, San Diego, CA, USA) was used to detect the presence of PIK3CA somatic mutations; samples were classified as positive or negative for PIK3CA mutations based on their presence or absence. For determining mRNA levels of HER2, total RNA extracted from FFPE tumor samples was assayed on the nCounter platform (NanoString Technologies, Seattle, WA) using the nCounter Virtual Breast Cancer Estrogen Receptor Gene probe set comprising 196 breast cancer and estrogen receptor signaling pathway test genes and six internal reference genes.

\section{Statistical analysis}

Preplanned statistical analyses were performed on the analysis population, defined as patients who met all eligibility criteria and had available data for at least one of the 3 biomarkers, using PASW Statistics ver. 18 (SPSS Inc., Chicago, IL). A sample size of 500 patients was originally planned. For the primary objective, sample size calculations were based on the precision of the estimated prevalence of the three biomarkers (presence/absence of p95HER2 protein expression, PTEN deletion/ down-regulation, and PIK3CA mutation). The sample size had to provide enough biomarker-positive patients for the confidence intervals (CIs) to be sufficiently narrow. Using the large-sample normal approximation, a sample size of 500 would ensure that a two-sided $90 \% \mathrm{CI}$ for the p95HER2-positive rate would extend $\leq 0.04$ from the observed proportion. The sample size was further refined using power analysis because the primary objective included hazard ratio estimation. At the 5\% significance level and with $90 \%$ power, 68 progression or death events were needed to detect a hazard ratio of $\leq 0.45$ (biomarker-positive group relative to -negative group). All statistical tests were two-sided unless otherwise specified.

PFS lapatinib-based treatment or trastuzumab-based treatment was analyzed for the overall study population and for subgroups based on positive and negative biomarker status. Survival times and PFS-prognostic factor relationship were estimated using the Kaplan-Meier product-limit method and compared across subgroups using log-rank tests. RR was measured as the proportion of patients showing either $C R$ or PR to lapatinib-based treatment received post-study entry. For OS, survival outcomes were determined based on patient status at data cutoff (alive, deceased, or unknown; time to death was censored at the time of last contact for patients who were alive at the end of study or lost to follow-up), and analyzed using the Kaplan-Meier method.

Distributions of the three biomarkers were summarized using counts and percentages of patients who were positive or negative for p95HER2 expression, PTEN deletion/downregulation, and PIK3CA mutation at primary diagnosis, and at recurrence / metastasis, where available. A chi-square test, or a Fisher exact test for small frequency counts, was used to test for statistically significant associations among the three biomarkers. Multivariate Cox proportional hazards models were used to evaluate associations between status of the three biomarkers and PFS, on either lapatinib or trastuzumab after adjusting for relevant prognostic factors. No multiplicity adjustment was performed to control for type I error. In addition to the expression levels of the three biomarkers, other prognostic factors assessed for PFS with lapatinibbased treatment were HER2 fluorescence in situ hybridization-positivity at primary diagnosis and IHC $2+$ status at study entry. Prognostic factors analyzed for PFS with trastuzumab-based treatment included ethnic group and recurrence in the contralateral breast at study entry.

Conversion and conservation rates for the three biomarkers between primary diagnosis and recurrence/metastasis were determined by cross-tabulating data for patients with conservation or conversion (negative to positive or vice versa) of biomarker status. Associations between tumor mRNA markers and PFS on lapatinib-based treatment were evaluated using the Kaplan-Meier method. The differences in PFS between subgroups based on mRNA marker status were tested using the log-rank test.

\section{Ethical statement}

This study was performed in compliance with Good Clinical Practices and GlaxoSmithKline Standard Operating Procedures for all processes involved, including the archiving of essential documents. This study complies with US 21 CFR 312.120, as described in the Ethics and Good Clinical Practice section. This study was conducted in accordance with the International Conference on Harmonization (ICH) of Technical Requirements for Registration of Pharmaceuticals for Human Use Good Clinical Practice (GCP) and applicable country-specific requirements, all applicable subject privacy requirements, and the ethical principles outlined in the Declaration of Helsinki 2008. The study was monitored in accordance with ICH E6, Section 5.18.

The protocol, any amendments, the informed consent form, and other information that required pre-approval were reviewed and approved by the respective investigational center ethics committees and institutional review boards (IRBs), in accordance with the ICH of Technical Requirements for Registration of Pharmaceuticals for Human Use GCP and applicable country-specific requirements. Ethics committee and IRB approvals were maintained in the Sponsor's study file.

This study was conducted in accordance with ICH GCP and all applicable subject privacy requirements, and, the ethical principles outlined in the Declaration of Helsinki 2008. The study was monitored in accordance with ICH E6, Section 
Table 1. Patient demographics and baseline disease characteristics

\begin{tabular}{|c|c|}
\hline Characteristic & No. $(\%)(\mathrm{n}=154)$ \\
\hline \multicolumn{2}{|c|}{ Age distribution at primary diagnosis (yr) } \\
\hline Mean \pm SD & $48.1 \pm 9.8$ \\
\hline Median (range) & $52.9(24.8-76.5)$ \\
\hline$<50$ yr vs. $\geq 50$ yr & $85(55.2): 69(44.8)$ \\
\hline \multicolumn{2}{|c|}{ Age distribution at study entry (yr) } \\
\hline Mean \pm SD & $52.4 \pm 9.9$ \\
\hline Median (range) & $48.7(28.3-86.0)$ \\
\hline$<50$ yr vs. $\geq 50$ yr & $62(40.3): 92(59.7)$ \\
\hline \multicolumn{2}{|c|}{$\begin{array}{l}\text { Time between primary diagnosis } \\
\text { and study entry (yr) }\end{array}$} \\
\hline Mean \pm SD & $4.3 \pm 3.2$ \\
\hline Median (range) & $3.3(0.2-19.9)$ \\
\hline \multicolumn{2}{|l|}{ Postmenopausal } \\
\hline At primary diagnosis & $78(50.6)$ \\
\hline At study entry & $131(85.1)$ \\
\hline \multicolumn{2}{|l|}{ ECOG PS } \\
\hline 0 & $42(27.3)$ \\
\hline 1 & $107(69.5)$ \\
\hline 2 & $5(3.2)$ \\
\hline \multicolumn{2}{|l|}{ Ethnicity } \\
\hline Korean & $110(71.4)$ \\
\hline Chinese & $40(26.0)$ \\
\hline Filipino & $2(1.3)$ \\
\hline Malay & $2(1.3)$ \\
\hline \multicolumn{2}{|l|}{ Cancer stage $(s)^{a}$} \\
\hline \multicolumn{2}{|l|}{ At primary diagnosis } \\
\hline I, II, or III & $118(76.7)$ \\
\hline IV (de novo) & $34(22.1)$ \\
\hline Unknown & $2(1.3)$ \\
\hline \multicolumn{2}{|l|}{ At study entry ${ }^{b}$} \\
\hline I, II, or III (metastatic) & $2(1.3)$ \\
\hline IV (de novo) & $150(97.4)$ \\
\hline Unknown & 0 \\
\hline \multicolumn{2}{|l|}{ HER2 status } \\
\hline \multicolumn{2}{|l|}{ At primary diagnosis } \\
\hline Positive & 147 (95.5) \\
\hline Negative & $2(1.3)$ \\
\hline Not performed & $5(3.2)$ \\
\hline \multicolumn{2}{|l|}{ At study entry } \\
\hline Positive & $45(29.2)$ \\
\hline Negative & 0 \\
\hline Not done & $109(70.8)$ \\
\hline
\end{tabular}

(Continued)

\subsection{8 .}

Investigators were trained to conduct the study in accordance with GCPs and the protocol as defined in ICH E3, Section 9.6. Written commitments were obtained from inves-
Table 1. Continued

\begin{tabular}{lc} 
Characteristic & No. $(\%)(\mathbf{n}=\mathbf{1 5 4})$ \\
ER status & \\
At primary diagnosis & \\
$\quad$ Positive & $65(42.2)$ \\
Negative & $85(55.2)$ \\
Unknown & $4(2.6)$ \\
At study entry & \\
Positive & $20(13.0)$ \\
Negative & $22(14.3)$ \\
Unknown & $112(72.7)$ \\
\hline
\end{tabular}

SD, standard deviation; ECOG PS, Eastern Cooperative Oncology Group performance status; HER2, human epidermal growth factor receptor 2; ER, estrogen receptor. a)According to the American Joint Committee on Cancer 7 th edition, ${ }^{\text {b) }}$ Data were not recorded for all patients.

tigators to comply with GCP and to conduct the study in accordance with the protocol.

Written informed consent was obtained from each subject prior to the performance of any study-specific procedures. The investigator provided the subject with as much time as necessary to review the document, to inquire about details of the study, and to decide whether or not to participate in the study. The informed consent was signed and dated by the study subject and by the person who conducted the informed consent discussion. Case report forms were provided for each subject's data to be recorded.

\section{Results}

\section{Patient demographics and disease characteristics}

This study enrolled patients initiating lapatinib-based treatment for metastatic disease between August 2010 and December 2012. Of the enrolled 156 eligible patients, data for 154 were analyzed. Two patients from a single center in Malaysia were not included in the analysis because patient data could not be verified following the death of the principal investigator at that center. The median age of patients at primary diagnosis was 52.9 years and the median time between primary diagnosis and study entry was 3.3 years (Table 1). Most patients were either Korean $(71.4 \%)$ or Chinese $(26.0 \%)$. Biopsy or tumor resection was performed for all 154 patients in the analysis population at the time of primary diagnosis. At primary diagnosis, most patients $(76.7 \%)$ 
Table 2. Agents used in systemic therapy regimens

\begin{tabular}{|c|c|c|c|}
\hline Regimen number & Trastuzumab & Lapatinib & Taxane \\
\hline \multicolumn{4}{|l|}{ Prior to study entry ${ }^{b)}$} \\
\hline 1 (adjuvant; $\mathrm{n}=129$ ) & $87(67.4)$ & $2(1.6)$ & $85(65.9)$ \\
\hline 2 (metastatic first-line; $\mathrm{n}=82$ ) & $22(26.8)$ & $9(11.0)$ & $16(19.5)$ \\
\hline$\geq 3$ (metastatic second-line or greater; $\mathrm{n}=100$ ) & $14(14.0)$ & $25(25.0)$ & $11(11.0)$ \\
\hline \multicolumn{4}{|l|}{ After study entryc) } \\
\hline $1(\mathrm{n}=24)$ & 0 & $24(100)$ & $1(4.2)$ \\
\hline $2(\mathrm{n}=50)$ & 0 & $48(96.0)$ & 0 \\
\hline$\geq 3(n=103)$ & $17(16.5)$ & $65(63.1)$ & $5(4.9)$ \\
\hline
\end{tabular}

had stages I to III, and $22.1 \%$ had de novo stage IV disease; nearly all (97.4\%) had MBC by study entry. Invasive ductal carcinoma was the most common histology at primary diagnosis $(96.8 \%)$. Of the 154 patients in the analysis population, $147(95.5 \%)$ were positive for HER2 at primary diagnosis, with five not determined $(3.2 \%)$ and two negative $(1.3 \%)$ (Table 1). At study entry, HER2 status was not determined for the majority of patients $(\mathrm{n}=109,70.8 \%)$; however, all who did $(n=45,29.2 \%)$ were HER2+ (Table 1$)$. The majority of samples available for biomarker analyses were from the primary tumor, and biomarker data from matched primary / metastasis samples were available in $<15 \%$ of patients.

Prior to study entry, 24 patients received adjuvant systemic treatment for early-stage disease, 89 received treatment in adjuvant and recurrent/metastatic disease settings, and 42 received for recurrent/metastatic disease only. Trastuzumab $(67.4 \%)$ and taxanes $(65.9 \%)$ were the most common agents in first-line regimens in the metastatic setting prior to study entry, followed by hormonal therapy (14.7\%) and anthracyclines $(10.9 \%) ; 27.1 \%$ received other chemotherapy. At study entry, $97 \%$ (72 of 74 patients) of first- and second-line regimens contained lapatinib, with or without other chemotherapy agents (Table 2).

\section{Clinical outcomes}

Median PFS with lapatinib-based regimens in the full study population was 33.3 weeks (95\% CI, 26.1 to 40.5 weeks) (Fig. 1A). The RR (CR+PR) for lapatinib-based regimens was 16.9\% (Table 3). Median OS with lapatinib-based regimens was 110.7 weeks (95\% CI, 87.4 to 134.0 weeks) for the analysis population. At data cut-off (August 31, 2013), 70 patients had died (45.5\%), 59 were alive (38.3\%), and 25 were lost to follow-up $(16.2 \%)$. Most deaths were attributed to disease pro- gression $(n=63,90.0 \%)$. Incidence of new brain metastases after study entry was $20 \%$.

\section{Biomarker analyses for lapatinib treatment}

Core biomarkers p95HER2, PTEN, and PIK3CA and exploratory marker HER2 mRNA level were evaluated for their correlation with clinical responsiveness to lapatinib-based treatment in the metastatic setting. In the analysis population, there were no significant associations between the three biomarkers (p95HER2, PTEN, and PIK3CA) and PFS with lapatinib-based treatment. Biomarker-based subgroups showed similar median PFS with substantially overlapping CIs (Fig. 1B-D). No statistically significant associations between RR and the status of the 3 biomarkers were observed (data not shown). Median OS with lapatinib-based treatment also was not significantly different for biomarker-positive and biomarker-negative groups. Multivariate Cox regression analyses showed no significant associations between any of the three biomarkers and PFS or OS with lapatinib-based treatment, with or without adjustment for prognostic factors. Exploratory univariate analyses showed no significant associations of HER2 mRNA levels and PFS with lapatinib-based treatment (data not shown).

\section{Biomarker analysis for other prior trastuzumab treat- ment}

Analysis of biomarker and covariate correlation with clinical response to previous trastuzumab-based treatment demonstrated no statistically significant correlations among the three biomarkers (p95HER2, PTEN, and PIK3CA) at primary diagnosis. Trastuzumab (67.4\%) was the most common systemic agent in first-line regimens prior to study entry. 
A
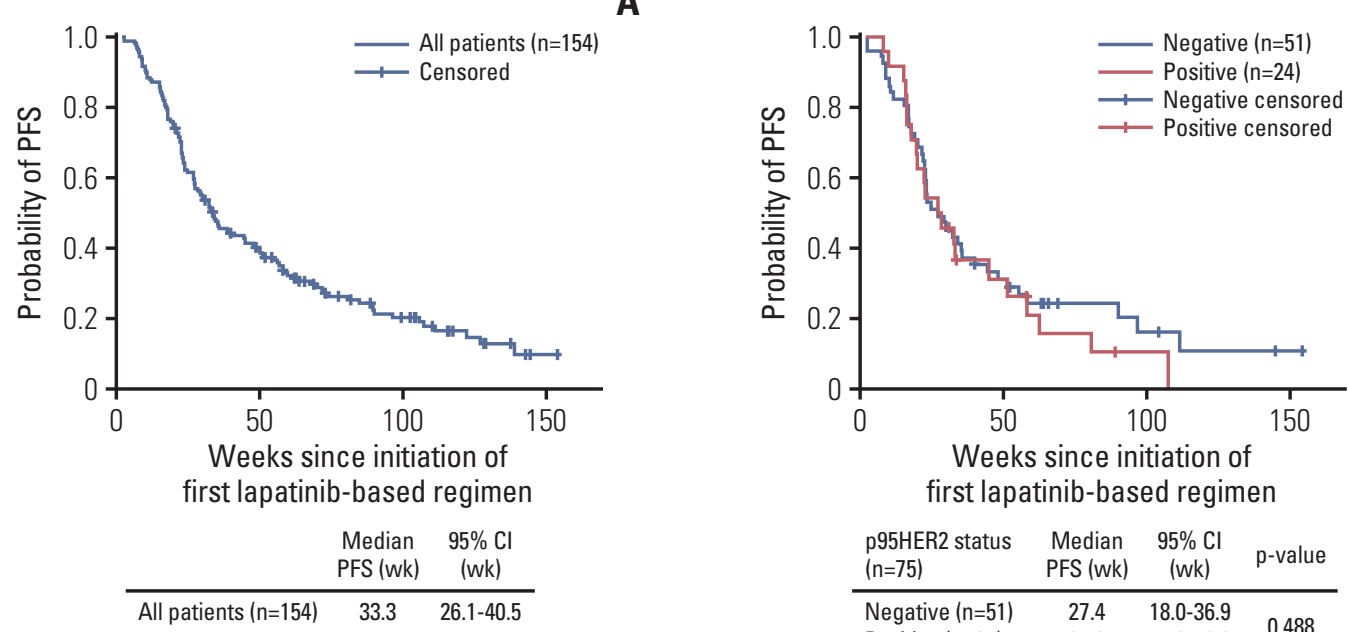

B

\begin{tabular}{lccc}
$\begin{array}{l}\text { p95HER2 status } \\
(n=75)\end{array}$ & $\begin{array}{c}\text { Median } \\
\text { PFS (wk) }\end{array}$ & $\begin{array}{c}95 \% \mathrm{Cl} \\
(w \mathrm{w})\end{array}$ & p-value \\
\hline Negative $(\mathrm{n}=51)$ & 27.4 & $18.0-36.9$ & 0.488 \\
Positive $(\mathrm{n}=24)$ & 27.6 & $15.2-40.0$ &
\end{tabular}

C
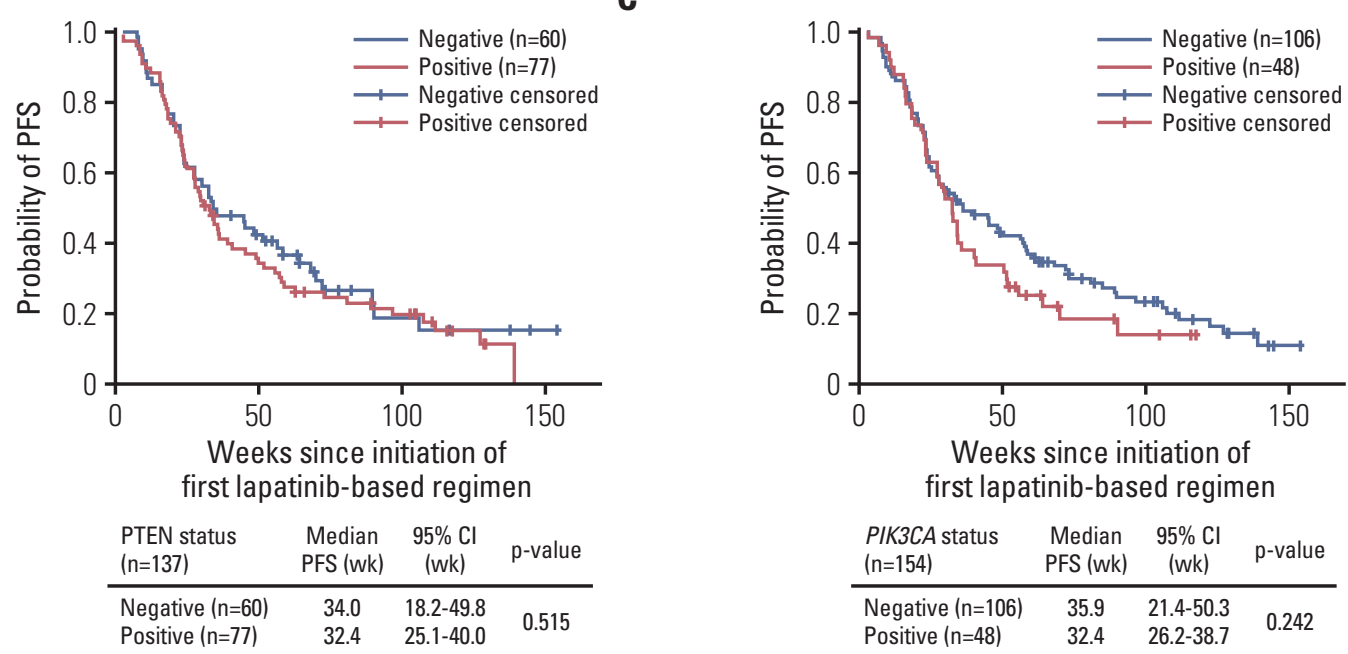

Fig. 1. Progression-free survival (PFS) with lapatinib based regimens for all patients and biomarker subgroups. (A) All patients. (B) Patients with p95HER2 expression. (C) Patients with PTEN deletion/down-regulation. (D) Patients with PIK3CA mutation. Note: Log-rank test of survival distribution equality for subgroups was defined by positive and negative biomarker status. CI, confidence interval.

Median PFS from initiation of the first-line trastuzumabbased regimen in the metastatic setting was 63.0 weeks $(95 \%$ CI, 53.6 to 72.4) (Fig. 2A). PFS with previous trastuzumabbased treatment in the metastatic setting had no statistically significant association with any of the three biomarkers. Accordingly, median PFS was similar in biomarker-positive and biomarker-negative subgroups (Fig. 2B-D). Multivariate analyses demonstrated that, of the prognostic factors tested, ethnic group and recurrence in the contralateral breast at study entry were the only potential prognostic factors associated with PFS with trastuzumab-based treatment; the strongest potential factor was Korean ethnicity (Table 4),
Table 3. RR with lapatinib-based regimens

\begin{tabular}{lc} 
Response & No. (\%) \\
CR & $6(3.9)$ \\
PR & $20(13.0)$ \\
Stable disease & $26(16.9)$ \\
Progressive disease & $86(55.8)$ \\
Unable to determine & $16(10.4)$ \\
Total & $154(100)$ \\
RR & $26(16.9)^{a)}$ \\
\hline
\end{tabular}

$\mathrm{RR}$, response rate; $\mathrm{CR}$, complete response; PR, partial res-

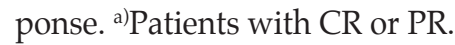


A
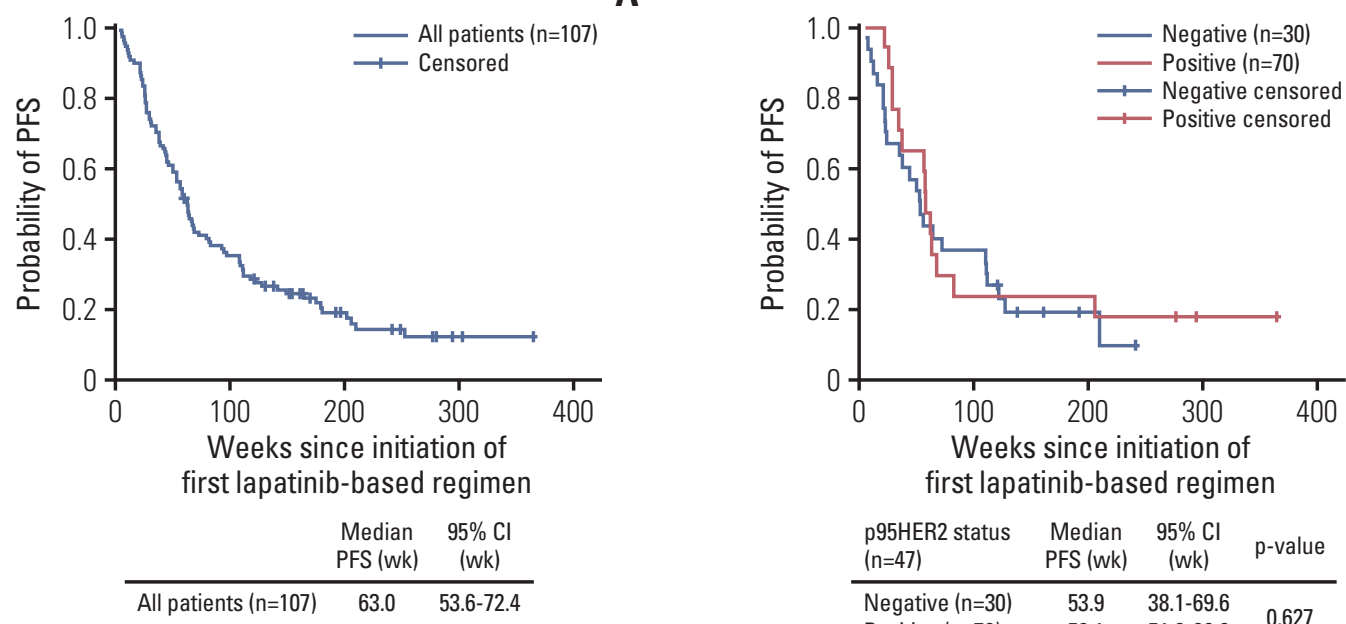

\begin{tabular}{|c|c|c|c|}
\hline $\begin{array}{l}\text { p95HER2 status } \\
(\mathrm{n}=47)\end{array}$ & $\begin{array}{l}\text { Median } \\
\text { PFS (wk) }\end{array}$ & $\begin{array}{c}95 \% \mathrm{Cl} \\
(w \mathrm{k})\end{array}$ & $\mathrm{p}$-value \\
\hline Negative $(n=30)$ & 53.9 & $38.1-69.6$ & 0.627 \\
\hline Positive $(n=70)$ & 59.1 & $51.6-66.6$ & 0.627 \\
\hline
\end{tabular}

C
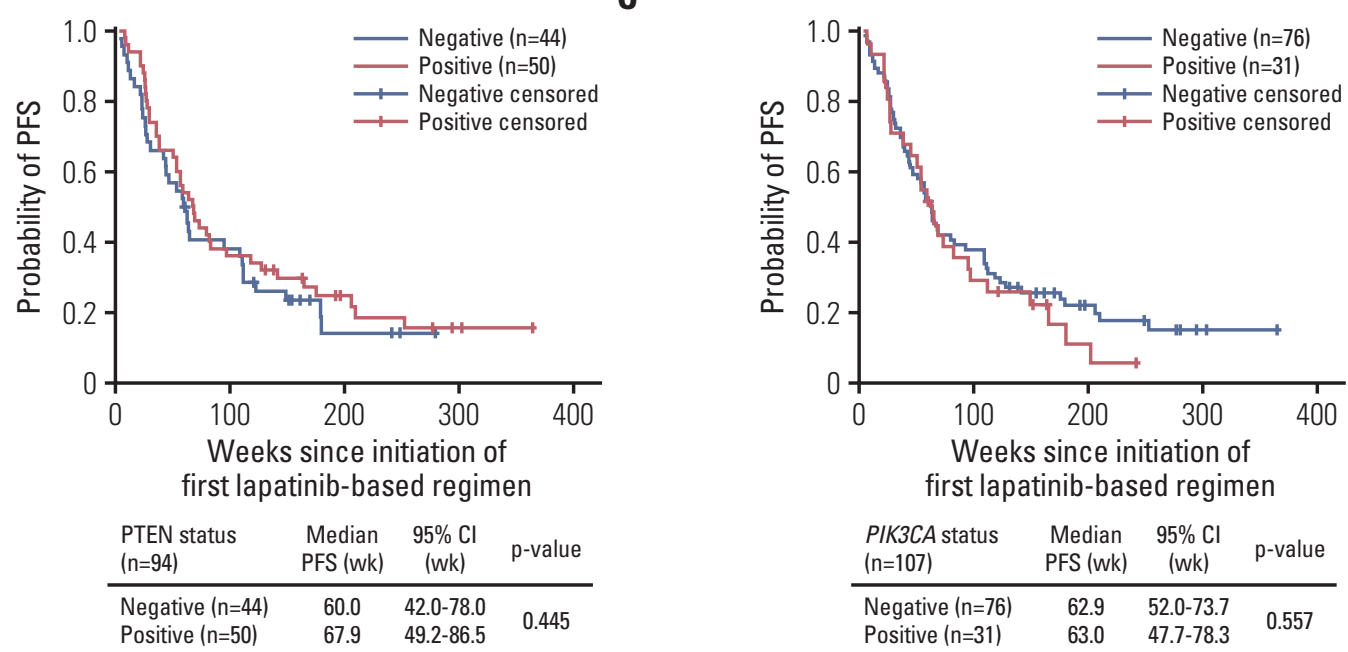

Fig. 2. Progression-fee survival (PFS) with trastuzumab-based regimens for all patients and biomarker subgroups. (A) All patients. (B) Patients with p95HER2 expression. (C) Patients with PTEN deletion/ down-regulation. (D) Patients with PIK3CA mutation. Note: Log-rank test of survival distribution equality for subgroups was defined by positive and negative biomarker status. CI, confidence interval.

possibly reflecting regional variations in treatment practices. Results from the multivariate analysis should, however, be interpreted cautiously as adjusted hazard ratios did not reach statistical significance at nominal p-value of 0.05 .

\section{Conservation/conversion rates}

Biomarker analyses demonstrated that the proportion of tumors positive for p95HER2 expression, PIK3CA mutation, and PTEN deletion/down-regulation at primary diagnosis were $32 \%, 31.2 \%$, and $56.2 \%$, respectively (Fig. 3). For patients with available tumor samples from primary diagnosis and re-biopsy, biomarker status remained $100 \%$ conserved for p95HER2 and mostly unchanged for PIK3CA (84.2\%). Conversely biomarker status was $62.5 \%$ conserved for PTEN (Fig. 4). However, the number of paired samples available for this analysis was very small (fewer than 20 for all 3 biomarkers), and these results should be interpreted with caution. 
Table 4. Potential prognostic factors affecting PFS with trastuzumab

\begin{tabular}{|c|c|c|c|c|}
\hline & Unadjusted HR (95\% CI) ${ }^{\mathrm{a})}$ & p-value ${ }^{\text {b) }}$ & Adjusted HR (95\% CI) & p-value ${ }^{c}$ \\
\hline p95HER2 expression & $0.85(0.44-1.60)$ & 0.628 & $1.01(0.45-2.20)$ & 0.975 \\
\hline PTEN deletion/ down-regulation & $0.84(0.53-1.30)$ & 0.456 & $1.30(0.60-3.00)$ & 0.488 \\
\hline PIK3CA mutation & $1.20(0.75-1.90)$ & 0.476 & $1.20(0.50-2.70)$ & 0.649 \\
\hline Ethnic group: Malayd) & $2.90(0.70-12.70)$ & 0.149 & $3.70(0.70-19.80)$ & 0.121 \\
\hline Ethnic group: Korean d) & $2.10(1.30-3.50)$ & 0.004 & $2.00(0.99-4.10)$ & 0.051 \\
\hline Contralateral breast & $0.37(0.14-0.99)$ & 0.047 & $0.30(0.08-1.10)$ & 0.078 \\
\hline
\end{tabular}

PFS, progression-free survival; HR, hazard ratio; CI, confidence interval; p95HER2, truncated human epidermal growth factor receptor 2; PTEN, phosphatase and tensin homolog; PIK3CA, phosphatidylinositol 3 kinase (catalytic subunit). ${ }^{\text {a) Uni- }}$

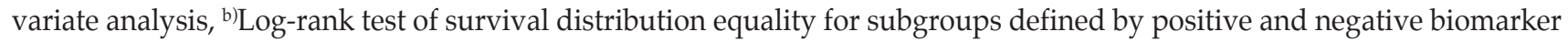
status, ${ }^{c}$ From Cox proportional hazards model adjusted for prognostic factors that are found to be significantly related to PFS on lapatinib in the univariable analysis for primary endpoint and 3 biomarkers (p95HER2 protein expression, PTEN deletion/downregulation, and PIK3CA mutation), d) Reference group: Chinese.

A

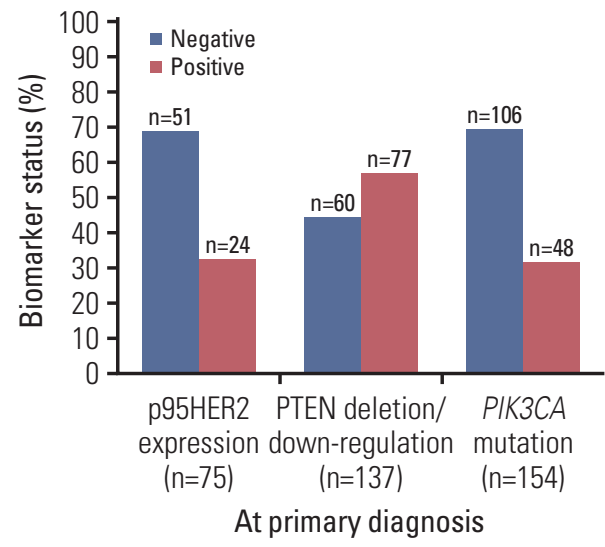

B

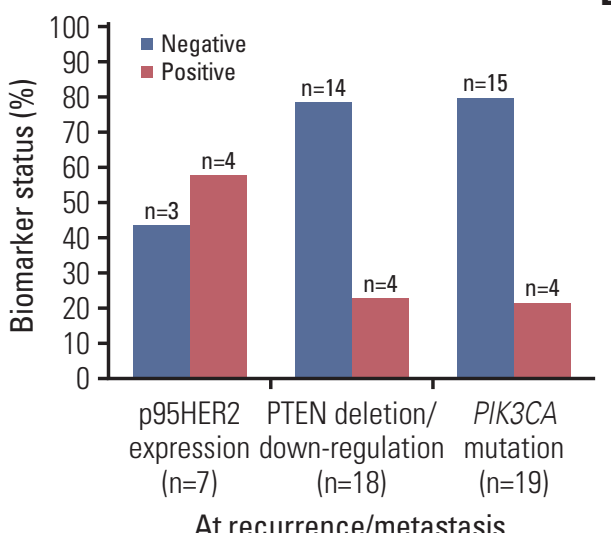

At recurrence/metastasis

Fig. 3. Biomarker status for the three biomarkers, at primary diagnosis (A) and at recurrence/metastasis (B), for the analysis population.

\section{Discussion}

Personalized cancer medicine based on molecular characteristics of a tumor from an individual patient and validated biomarkers with acceptable sensitivity and specificity has great potential in the therapy of many types of cancer [37]. Recent studies have identified tumor-infiltrating lymphocytes [38], genetic signatures [39], and hormone receptor status [40] as potential biomarkers for response to trastuzumab-based treatment. Limited understanding of biomarker profiles that can reliably predict response to lapatinib-based treatment prompted us to investigate biomarker profiles of p95HER2, PTEN, PIK3CA, and tumor HER2 mRNA levels in a population of lapatinib-treated HER2+ MBC patients from four Asian countries, many of whom were previously treated

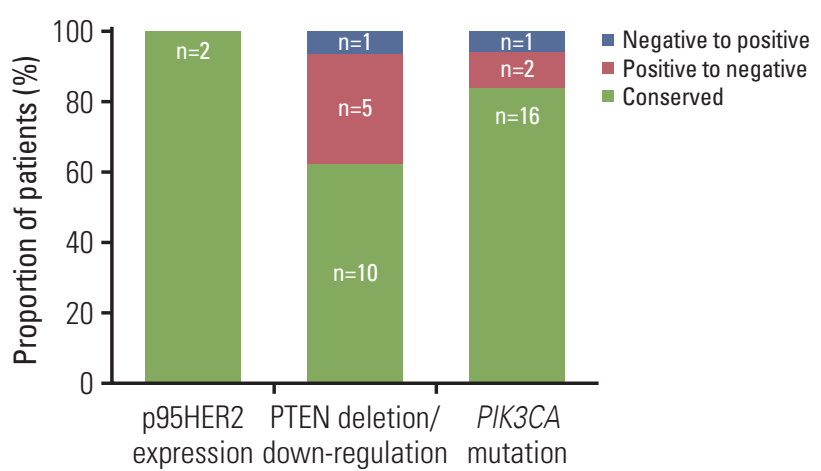

Fig. 4. Conversion/ conservation of biomarker status. 
with trastuzumab-based therapy. Our study shows that p95HER2, PTEN, and PIK3CA biomarker status was not predictive of PFS on either lapatinib-based or trastuzumab-based therapy in the metastatic setting. The status of the three biomarkers also was not predictive for CR, PR, or OS with lapatinib-based regimens. These results are in accordance with recent data for lapatinib-based treatment in relation to p95HER2 status in Korean patients [41], and to PIK3CA status in Chinese patients [17]. Our study did not reveal any PFS differences with trastuzumab-based treatment between PIK3CA WT and mutated subgroups, possibly because of the small sample size. However, PFS was shorter in patients with PIK3CA mutations in the CLEOPATRA trial [31].

PIK3CA mutations have been associated with less response to chemotherapy and the combination of chemotherapy and HER2-targeted agents in several neoadjuvant trials [42]. However, these results do not indicate a direct link between PIK3CA mutation status and response to HER2-targeted agents because patients received chemotherapy concomitantly. Randomized trials in the adjuvant setting have reported that alterations in PIK3CA or PTEN do not predict trastuzumab resistance $[43,44]$. In addition, several reports have demonstrated lack of association between PIK3CA mutations or PTEN loss, and the efficacy of lapatinib, regardless of whether administered alone or in combination with chemotherapy [34,35,45,46]. Moreover, patients with HER2overexpressing inflammatory breast cancers responded to lapatinib monotherapy regardless of PTEN status [47]. Thus the predictive performance of the three biomarkers, p95HER2, PTEN, and PIK3CA in our analysis mirrors results from other global studies. Furthermore, we show that PFS with lapatinib-based treatment in Asian patients is consistent with that reported previously in real-world settings [17,48-50]. In the GeparQuattro study, response to trastuzumab-based therapy was found to correlate with HER2 mRNA levels only in patients with estrogen receptor-positive tumors [28]. Similarly, in the analogous, albeit smaller, trastuzumab arm of the NeoALTTO study, HER2 protein expression trended toward a correlation with pathologic complete response (pCR) in the hormone receptor-positive group, but there was no relationship between HER2 and pCR in the hormone receptor-negative group [51]. However, HER2 tumor mRNA levels were not predictive of PFS on lapatinib-based treatment in our study.

In our study, p95HER2 expression and PTEN deletion/ down-regulation at primary diagnosis were $32 \%$, and $56.2 \%$, respectively, consistent with earlier reports wherein the frequency of p95HER2 expression was approximately $30 \%$ in HER2+ tumors $[52,53]$ and approximately $50 \%$ were PTENdeficient [54]. Furthermore, the frequency of PIK3CA mutation was 39\% in an array of HER2+ primary breast cancers [55], akin to the $31 \%$ PIK3CA mutation frequency at primary diagnosis in our study. Importantly, although a number of different methods were used to measure individual biomarkers across these studies and no standard validated method exists thus far, the results are very similar overall.

Although performed in a largely Asian patient population, mostly of Korean and Chinese ethnicity, with HER2+ ABC, our results are consistent with global studies. Thus, our results are not likely to be biased or population-specific, and may have relevance for other HER2+ patients with biomarker profiles of interest within the general breast cancer population. Interestingly, we found that incidence of new brain metastases after study entry was approximately $20 \%$, somewhat lower than the $25 \%$ to $36 \%$ range reported in previous studies of trastuzumab-based treatment in HER2+ breast cancer patients $[48-50,56]$. This is potentially encouraging in light of reports that lapatinib-capecitabine [57-59] and lapatinib-trastuzumab [60] combinations may provide clinical benefit in patients with $\mathrm{MBC}$ with brain metastases.

Our study was limited by tissue availability, repeat biopsies at metastasis in only 19 patients, and slow patient recruitment, which resulted in a substantially smaller analysis population $(n=154)$ than originally planned $(n=500)$. As can be expected in a non-interventional study, there was no randomization for treatment assignment, which differs from biomarker analyses from randomized trials such as NeoALTTO and GeparQuattro. Results from the analysis of biomarker conservation/ conversion patterns in our study should be interpreted carefully, keeping in mind the limited availability of paired samples. Finally, as our study allowed retrospective enrollment, precision of survival estimates may have been reduced because of unavailability of medical record information (e.g., dates of disease progression or death) for some of these patients.

In conclusion, our study did not demonstrate any significant correlation between biomarker status of p95HER2, PTEN deletion/ downregulation, PI3KCA mutation or HER2 mRNA levels and clinical outcome on lapatinib-based treatment in the enrolled cohort of HER2+ ABC patients, similar to previous reports. Additionally, median PFS on lapatinibbased treatment in our study was similar to that observed in previous studies of lapatinib-based treatment in $\mathrm{ABC} / \mathrm{MBC}$ $[17,48-50]$. Predictive biomarkers can facilitate the identification and establishment of individualized treatment for patients with HER2+ ABC. The correlations between biomarkers and response/resistance to lapatinib-based treatment have not been fully delineated thus far. Hence, additional studies are needed to better identify patient populations likely to benefit from lapatinib-based treatment for HER2+ ABC. 


\section{Conflicts of Interest}

Sung-Bae Kim has received funding from Novartis, Sanofi Aventis, Kyowa Kirim Inc., Dongkook Pharmaceuticals Company Ltd. Gerardo Cornelio has received funding from Renovo; has served as a consultant/advisory role for Astra Zeneca Oncology, BF, MSD Oncology, and Esai Oncology. Yoon Sim Yap has served as a consultant/advisory role from Novartis. Soonmyung Paik has received funding from GSK for this study; has served as a consultant/advisory role for Leica Biosystems. Christos Nathaniel is an employee of Novartis Pharmaceuticals Corporation. The following authors have nothing to disclose: In-Gu Do, Janice Tsang, Tae-You Kim, Gyungyub Gong, Suee Lee, Ting-Ying Ng, Sarah Park, Ho-Suk Oh, Joanne Chiu, Joohyuk Sohn, Moonhee Lee, Young-Jin Choi, Eun Mi Lee, Kyong-Hwa Park, and Jungsil Ro.

\section{Acknowledgments}

This study was sponsored by GlaxoSmithKline. Lapatinib is an asset of Novartis Pharmaceuticals Corporation as of March 2, 2015. Financial support for medical editorial assistance was provided by Novartis Pharmaceuticals. We thank the pathologists for their work analyzing the tumor samples for this manuscript: Hee Jin Lee. We thank Maupali Dasgupta, PhD, ProEd Communications, Inc., for her medical editorial assistance with this manuscript.

\section{Author Details}

${ }^{1}$ Department of Oncology, Asan Medical Center, University of Ulsan College of Medicine, Seoul, ²Department of Pathology, Kangbuk Samsung Hospital, Sungkyunkwan University School of Medicine, Seoul, Korea, ${ }^{3}$ Department of Medicine, Queen Mary Hospital, The University of Hong Kong, Hong Kong, China, ${ }^{4}$ Department of Internal Medicine, Seoul National University Hospital, Seoul, Korea, ${ }^{5}$ Division of Medical Oncology, National Cancer Centre Singapore, Singapore, ${ }^{6}$ Department of Medicine, San Juan De Dios Hospital, Manila, Philippines, 'Department of Pathology, Asan Medical Center, University of Ulsan College of Medicine, Seoul, ${ }^{8}$ Department of Medical Oncology and Severance Biomedical Research Institute, Yonsei University College of Medicine, Seoul, 'Department of Internal Medicine, Dong-A University Hospital, Busan, Korea, ${ }^{10}$ Department of Clinical Oncology, Tuen Mun Hospital, Hong Kong, China, ${ }^{11}$ The Center for Anti-Cancer Companion Diagnostics, Bio-MAX/ N-Bio, Seoul National University, Seoul, ${ }^{12}$ Department of Hematology-Oncology, Gangneung Asan Hospital, University of Ulsan College of Medicine, Gangneung, ${ }^{13}$ Division of Medical Oncology, Yonsei Cancer Center, Seoul, ${ }^{14}$ Division of Hematology-Oncology, Inha University Hospital, Incheon, ${ }^{15}$ Department of HematologyOncology, Pusan National University Hospital, Busan, ${ }^{16}$ Department of Internal Medicine, Kosin University Gospel Hospital, Busan, ${ }^{17}$ Department of Internal Medicine, Korea University Anam Hospital, Seoul, Korea, ${ }^{18}$ Novartis Pharmaceuticals Corporation, APSA, Midrand, South Africa, ${ }^{19}$ Graduate School of Cancer Science and Policy, National Cancer Center, Goyang, Korea

\section{References}

1. Youlden DR, Cramb SM, Yip CH, Baade PD. Incidence and mortality of female breast cancer in the Asia-Pacific region. Cancer Biol Med. 2014;11:101-15.

2. Shin HR, Joubert C, Boniol M, Hery C, Ahn SH, Won YJ, et al. Recent trends and patterns in breast cancer incidence among Eastern and Southeastern Asian women. Cancer Causes Control. 2010;21:1777-85.

3. Katanoda K, Matsuda T, Matsuda A, Shibata A, Nishino Y, Fujita M, et al. An updated report of the trends in cancer incidence and mortality in Japan. Jpn J Clin Oncol. 2013;43:492507.

4. Jung KW, Won YJ, Kong HJ, Oh CM, Seo HG, Lee JS. Cancer statistics in Korea: incidence, mortality, survival and prevalence in 2010. Cancer Res Treat. 2013;45:1-14.

5. Burstein HJ. The distinctive nature of HER2-positive breast cancers. N Engl J Med. 2005;353:1652-4.

6. Slamon DJ, Leyland-Jones B, Shak S, Fuchs H, Paton V, Bajamonde A, et al. Use of chemotherapy plus a monoclonal antibody against HER2 for metastatic breast cancer that overex- presses HER2. N Engl J Med. 2001;344:783-92.

7. Slamon D, Eiermann W, Robert N, Pienkowski T, Martin M, Press M, et al. Adjuvant trastuzumab in HER2-positive breast cancer. N Engl J Med. 2011;365:1273-83.

8. Romond EH, Perez EA, Bryant J, Suman VJ, Geyer CE Jr, Davidson NE, et al. Trastuzumab plus adjuvant chemotherapy for operable HER2-positive breast cancer. N Engl J Med. 2005; 353:1673-84

9. Piccart-Gebhart MJ, Procter M, Leyland-Jones B, Goldhirsch A, Untch M, Smith I, et al. Trastuzumab after adjuvant chemotherapy in HER2-positive breast cancer. N Engl J Med. 2005;353:1659-72.

10. Gianni L, Eiermann W, Semiglazov V, Manikhas A, Lluch A, Tjulandin S, et al. Neoadjuvant chemotherapy with trastuzumab followed by adjuvant trastuzumab versus neoadjuvant chemotherapy alone, in patients with HER2-positive locally advanced breast cancer (the NOAH trial): a randomised controlled superiority trial with a parallel HER2-negative cohort. Lancet. 2010;375:377-84. 
11. Cardoso F, Senkus E, Costa A, Papadopoulos E, Aapro M, Andre F, et al. 4th ESO-ESMO International Consensus Guidelines for Advanced Breast Cancer (ABC 4). Ann Oncol. 2018; 29:1634-57.

12. Giordano SH, Temin S, Chandarlapaty S, Crews JR, Esteva FJ, Kirshner JJ, et al. Systemic therapy for patients with advanced human epidermal growth factor receptor 2-positive breast cancer: ASCO clinical practice guideline update. J Clin Oncol. 2018;36:2736-40

13. National Comprehensive Cancer Network. NCCN clinical practice guidelines in oncology: breast cancer, version 1.2018 [Internet]. Plymouth Meeting, PA: National Comprehensive Cancer Network; 2018 [cited 2018 Sep 7]. Available from: http:// www.nccn.org/professionals/physician_gls/pdf/brea st.pdf.

14. Geyer CE, Forster J, Lindquist D, Chan S, Romieu CG, Pienkowski T, et al. Lapatinib plus capecitabine for HER2-positive advanced breast cancer. N Engl J Med. 2006;355:2733-43.

15. Guan Z, Xu B, DeSilvio ML, Shen Z, Arpornwirat W, Tong Z, et al. Randomized trial of lapatinib versus placebo added to paclitaxel in the treatment of human epidermal growth factor receptor 2-overexpressing metastatic breast cancer. J Clin Oncol. 2013;31:1947-53.

16. Iwata H, Fujii H, Masuda N, Mukai H, Nishimura Y, Katsura $\mathrm{K}$, et al. Efficacy, safety, pharmacokinetics and biomarker findings in patients with HER2-positive advanced or metastatic breast cancer treated with lapatinib in combination with capecitabine: results from 51 Japanese patients treated in a clinical study. Breast Cancer. 2015;22:192-200.

17. Xu BH, Jiang ZF, Chua D, Shao ZM, Luo RC, Wang XJ, et al. Lapatinib plus capecitabine in treating HER2-positive advanced breast cancer: efficacy, safety, and biomarker results from Chinese patients. Chin J Cancer. 2011;30:327-35.

18. Wang H. Lapatinib for the treatment of breast cancer in the People's Republic of China. Onco Targets Ther. 2014;7:136773.

19. Baselga J, Cortes J, Kim SB, Im SA, Hegg R, Im YH, et al. Pertuzumab plus trastuzumab plus docetaxel for metastatic breast cancer. N Engl J Med. 2012;366:109-19.

20. Santa-Maria CA, Nye L, Mutonga MB, Jain S, Gradishar WJ. Management of metastatic HER2-positive breast cancer: where are we and where do we go from here? Oncology (Williston Park). 2016;30:148-55.

21. Swain SM, Kim SB, Cortes J, Ro J, Semiglazov V, Campone M, et al. Pertuzumab, trastuzumab, and docetaxel for HER2-positive metastatic breast cancer (CLEOPATRA study): overall survival results from a randomised, double-blind, placebocontrolled, phase 3 study. Lancet Oncol. 2013;14:461-71.

22. Verma S, Miles D, Gianni L, Krop IE, Welslau M, Baselga J, et al. Trastuzumab emtansine for HER2-positive advanced breast cancer. N Engl J Med. 2012;367:1783-91.

23. Scaltriti M, Rojo F, Ocana A, Anido J, Guzman M, Cortes J, et al. Expression of p95HER2, a truncated form of the HER2 receptor, and response to anti-HER2 therapies in breast cancer. J Natl Cancer Inst. 2007;99:628-38.

24. Scaltriti M, Chandarlapaty S, Prudkin L, Aura C, Jimenez J,
Angelini PD, et al. Clinical benefit of lapatinib-based therapy in patients with human epidermal growth factor receptor 2-positive breast tumors coexpressing the truncated p95HER2 receptor. Clin Cancer Res. 2010;16:2688-95.

25. Kataoka Y, Mukohara T, Shimada H, Saijo N, Hirai M, Minami $\mathrm{H}$. Association between gain-of-function mutations in PIK3CA and resistance to HER2-targeted agents in HER2-amplified breast cancer cell lines. Ann Oncol. 2010;21:255-62.

26. Nagata Y, Lan KH, Zhou X, Tan M, Esteva FJ, Sahin AA, et al. PTEN activation contributes to tumor inhibition by trastuzumab, and loss of PTEN predicts trastuzumab resistance in patients. Cancer Cell. 2004;6:117-27.

27. Paik S, Kim C, Wolmark N. HER2 status and benefit from adjuvant trastuzumab in breast cancer. N Engl J Med. 2008; 358:1409-11.

28. Denkert C, Huober J, Loibl S, Prinzler J, Kronenwett R, DarbEsfahani S, et al. HER2 and ESR1 mRNA expression levels and response to neoadjuvant trastuzumab plus chemotherapy in patients with primary breast cancer. Breast Cancer Res. 2013; 15:R11.

29. Esteva FJ, Guo H, Zhang S, Santa-Maria C, Stone S, Lanchbury JS, et al. PTEN, PIK3CA, p-AKT, and p-p70S6K status: association with trastuzumab response and survival in patients with HER2-positive metastatic breast cancer. Am J Pathol. 2010; 177:1647-56.

30. Razis E, Bobos M, Kotoula V, Eleftheraki AG, Kalofonos HP, Pavlakis K, et al. Evaluation of the association of PIK3CA mutations and PTEN loss with efficacy of trastuzumab therapy in metastatic breast cancer. Breast Cancer Res Treat. 2011; 128:447-56.

31. Baselga J, Cortes J, Im SA, Clark E, Ross G, Kiermaier A, et al. Biomarker analyses in CLEOPATRA: a phase III, placebo-controlled study of pertuzumab in human epidermal growth factor receptor 2-positive, first-line metastatic breast cancer. J Clin Oncol. 2014;32:3753-61.

32. Baselga J, Lewis Phillips GD, Verma S, Ro J, Huober J, Guardino AE, Samant MK, et al. Relationship between tumor biomarkers and efficacy in EMILIA, a phase III study of trastuzumab emtansine in HER2-positive metastatic breast cancer. Clin Cancer Res. 2016;22:3755-63.

33. Berns K, Horlings HM, Hennessy BT, Madiredjo M, Hijmans $\mathrm{EM}$, Beelen $\mathrm{K}$, et al. A functional genetic approach identifies the PI3K pathway as a major determinant of trastuzumab resistance in breast cancer. Cancer Cell. 2007;12:395-402.

34. Toi M, Iwata H, Fujiwara Y, Ito Y, Nakamura S, Tokuda Y, et al. Lapatinib monotherapy in patients with relapsed, advanced, or metastatic breast cancer: efficacy, safety, and biomarker results from Japanese patients phase II studies. Br J Cancer. 2009;101:1676-82.

35. Dave B, Migliaccio I, Gutierrez MC, Wu MF, Chamness GC, Wong $\mathrm{H}$, et al. Loss of phosphatase and tensin homolog or phosphoinositol-3 kinase activation and response to trastuzumab or lapatinib in human epidermal growth factor receptor 2-overexpressing locally advanced breast cancers. J Clin Oncol. 2011;29:166-73.

36. Duchnowska R, Sperinde J, Chenna A, Haddad M, Paquet A, 
Lie Y, et al. Quantitative measurements of tumoral p95HER2 protein expression in metastatic breast cancer patients treated with trastuzumab: independent validation of the p95HER2 clinical cutoff. Clin Cancer Res. 2014;20:2805-13.

37. Wistuba II, Gelovani JG, Jacoby JJ, Davis SE, Herbst RS. Methodological and practical challenges for personalized cancer therapies. Nat Rev Clin Oncol. 2011;8:135-41.

38. Luen SJ, Salgado R, Fox S, Savas P, Eng-Wong J, Clark E, et al. Tumour-infiltrating lymphocytes in advanced HER2-positive breast cancer treated with pertuzumab or placebo in addition to trastuzumab and docetaxel: a retrospective analysis of the CLEOPATRA study. Lancet Oncol. 2017;18:52-62.

39. Fumagalli D, Venet D, Ignatiadis M, Azim HA Jr, Maetens M, Rothe F, et al. RNA sequencing to predict response to neoadjuvant anti-HER2 therapy: a secondary analysis of the NeoALTTO randomized clinical trial. JAMA Oncol. 2017;3:227-34.

40. Park HS, Sohn J, Kim SI, Park S, Park HS, Gho SG, et al. Effects of hormone receptor status on the durable response of trastuzumab-based therapy in metastatic breast cancer. Breast Cancer Res Treat. 2017;163:255-62.

41. Han SW, Cha Y, Paquet A, Huang W, Weidler J, Lie Y, et al. Correlation of HER2, p95HER2 and HER3 expression and treatment outcome of lapatinib plus capecitabine in HER2positive metastatic breast cancer. PLoS One. 2012;7:e39943.

42. Loibl S, von Minckwitz G, Schneeweiss A, Paepke S, Lehmann A, Rezai M, et al. PIK3CA mutations are associated with lower rates of pathologic complete response to anti-human epidermal growth factor receptor 2 (HER2) therapy in primary HER2-overexpressing breast cancer. J Clin Oncol. 2014;32: 3212-20.

43. Loi S, Michiels S, Lambrechts D, Fumagalli D, Claes B, Kellokumpu-Lehtinen PL, et al. Somatic mutation profiling and associations with prognosis and trastuzumab benefit in early breast cancer. J Natl Cancer Inst. 2013;105:960-7.

44. Perez EA, Dueck AC, McCullough AE, Chen B, Geiger XJ, Jenkins RB, et al. Impact of PTEN protein expression on benefit from adjuvant trastuzumab in early-stage human epidermal growth factor receptor 2-positive breast cancer in the North Central Cancer Treatment Group N9831 trial. J Clin Oncol. 2013;31:2115-22.

45. Johnston S, Trudeau M, Kaufman B, Boussen H, Blackwell K, LoRusso $\mathrm{P}$, et al. Phase II study of predictive biomarker profiles for response targeting human epidermal growth factor receptor 2 (HER-2) in advanced inflammatory breast cancer with lapatinib monotherapy. J Clin Oncol. 2008;26:1066-72.

46. Xu B, Guan Z, Shen Z, Tong Z, Jiang Z, Yang J, et al. Association of phosphatase and tensin homolog low and phosphatidylinositol3-kinase catalytic subunit alpha gene mutations on outcome in human epidermal growth factor receptor 2-positive metastatic breast cancer patients treated with first-line lapatinib plus paclitaxel or paclitaxel alone. Breast Cancer Res. 2014;16:405.

47. Xia W, Husain I, Liu L, Bacus S, Saini S, Spohn J, et al. Lapatinib antitumor activity is not dependent upon phosphatase and tensin homologue deleted on chromosome 10 in ErbB2overexpressing breast cancers. Cancer Res. 2007;67:1170-5.
48. Cameron D, Casey M, Press M, Lindquist D, Pienkowski T, Romieu CG, et al. A phase III randomized comparison of lapatinib plus capecitabine versus capecitabine alone in women with advanced breast cancer that has progressed on trastuzumab: updated efficacy and biomarker analyses. Breast Cancer Res Treat. 2008;112:533-43.

49. Johnston S, Pippen J Jr, Pivot X, Lichinitser M, Sadeghi S, Dieras $\mathrm{V}$, et al. Lapatinib combined with letrozole versus letrozole and placebo as first-line therapy for postmenopausal hormone receptor-positive metastatic breast cancer. J Clin Oncol. 2009;27:5538-46.

50. Di Leo A, Gomez HL, Aziz Z, Zvirbule Z, Bines J, Arbushites MC, et al. Phase III, double-blind, randomized study comparing lapatinib plus paclitaxel with placebo plus paclitaxel as first-line treatment for metastatic breast cancer. J Clin Oncol. 2008;26:5544-52.

51. Scaltriti M, Nuciforo P, Bradbury I, Sperinde J, Agbor-Tarh D, Campbell C, et al. High HER2 expression correlates with response to the combination of lapatinib and trastuzumab. Clin Cancer Res. 2015;21:569-76.

52. Saez R, Molina MA, Ramsey EE, Rojo F, Keenan EJ, Albanell J, et al. p95HER-2 predicts worse outcome in patients with HER-2-positive breast cancer. Clin Cancer Res. 2006;12:424-31.

53. Sperinde J, Jin X, Banerjee J, Penuel E, Saha A, Diedrich G, et al. Quantitation of p95HER2 in paraffin sections by using a p95-specific antibody and correlation with outcome in a cohort of trastuzumab-treated breast cancer patients. Clin Cancer Res. 2010;16:4226-35.

54. Fujita T, Doihara H, Kawasaki K, Takabatake D, Takahashi H, Washio $\mathrm{K}$, et al. PTEN activity could be a predictive marker of trastuzumab efficacy in the treatment of ErbB2-overexpressing breast cancer. Br J Cancer. 2006;94:247-52.

55. Cancer Genome Atlas Network. Comprehensive molecular portraits of human breast tumours. Nature. 2012;490:61-70.

56. Clayton AJ, Danson S, Jolly S, Ryder WD, Burt PA, Stewart $\mathrm{AL}$, et al. Incidence of cerebral metastases in patients treated with trastuzumab for metastatic breast cancer. Br J Cancer. 2004;91:639-43.

57. Metro G, Foglietta J, Russillo M, Stocchi L, Vidiri A, Giannarelli D, et al. Clinical outcome of patients with brain metastases from HER2-positive breast cancer treated with lapatinib and capecitabine. Ann Oncol. 2011;22:625-30.

58. Ro J, Park S, Kim S, Kim TY, Im YH, Rha SY, et al. Clinical outcomes of HER2-positive metastatic breast cancer patients with brain metastasis treated with lapatinib and capecitabine: an open-label expanded access study in Korea. BMC Cancer. 2012;12:322.

59. Sutherland S, Ashley S, Miles D, Chan S, Wardley A, Davidson $\mathrm{N}$, et al. Treatment of HER2-positive metastatic breast cancer with lapatinib and capecitabine in the lapatinib expanded access programme, including efficacy in brain metastases: the UK experience. Br J Cancer. 2010;102:995-1002.

60. Yap YS, Cornelio GH, Devi BC, Khorprasert C, Kim SB, Kim TY, et al. Brain metastases in Asian HER2-positive breast cancer patients: anti-HER2 treatments and their impact on survival. Br J Cancer. 2012;107:1075-82. 